\title{
Birth Year
}

National Cancer Institute

\section{Source}

National Cancer Institute. Birth Year. NCI Thesaurus. Code C83164.

The year in which a person was born. 